\title{
Measuring Viscoelastic Properties of Living Cells
}

\author{
Yang $\mathrm{Bu}^{1} \quad$ Long $\mathbf{L i}^{1} \quad$ Chendong Yang ${ }^{1} \quad{\text { Rui } \mathbf{L i}^{1} \quad \text { Jizeng Wang }}^{1 \star}$ \\ $\left({ }^{1}\right.$ Key Laboratory of Mechanics on Disaster and Environment in Western China, Ministry of Education, \\ College of Civil Engineering and Mechanics, Lanzhou University, Lanzhou 730000, China)
}

Received 3 February 2019; revision received 5 April 2019; Accepted 16 June 2019;

published online 19 July 2019

(c) The Author(s) 2019

\begin{abstract}
Precise measurement of mechanical properties of living cells is important in understanding their mechanics-biology relations. In this study, we adopted the atomic force microscope to measure the creep deformation and stress relaxation of six different human cell lines. We examined whether the measured creep and relaxation trajectories satisfy a verification relation derived based on the linear viscoelastic theory. We compared the traditional spring-dashpot and the newly developed power-law-type constitutive relations in fitting the experimental measurements. We found that the human normal liver (L02), hepatic cancer (HepG2), hepatic stellate (LX2) and gastric cancer (NCI-N87) cell lines are linear viscoelastic materials, and human normal gastric (GES-1) and gastric cancer (SGC7901) cell lines are nonlinear due to failing in satisfying the verification relation for linear viscoelastic theory. The three-parameter power-law-type constitutive relation can fit the experimental measurements better than that of the five-parameter classical spring-dashpot.
\end{abstract}

KEY WORDS Atomic force microscope, Living cells, Linear viscoelasticity, Creep, Relaxation

\section{Introduction}

Living cells are soft and complex materials with cytoskeletons continuously subjected to highly dynamic remodeling. Their mechanical properties have been found to be related to various cellular physiology behaviors, including cell locomotion [1], differentiation [2], adhesion [3, 4] and nanoparticle endocytosis [5-7]. In addition, it has been widely revealed that many human diseases also have close relationships with the mechanical properties of corresponding cells [8-13]. For example, the malariainfected red blood cells can become stiffer and sticky, which are unfavorable for the oxygen transport, eventually leading to severe anemia, coma or even death [12]. Therefore, precise measurement of the mechanical properties of living cells can be very crucial for diagnosing human disease and better understanding biological processes.

Quantitative measurement of material properties of living cells is usually difficult due to the facts of their low stiffness, small size and severe thermal fluctuations. Despite these difficulties, a series of experimental efforts have been conducted to probe the mechanical properties of living cells by using various techniques, including the atomic force microscopy (AFM) [14-18], magnetic twisting cytometry [19], magnetic or optical tweezers [20], microplate rheometer [21] and particle tracking microrheology $[22,23]$. In these measurements, indentation techniques based on the AFM were most popularly adopted. As long as the force-indentation curves are measured by using the AFM, the

\footnotetext{
* Corresponding author. E-mail: jzwang@lzu.edu.cn
} 
Hertz contact calibration model based on the isotropic elastic theory is usually introduced to fit them. Material constants of cells are determined as fitting parameters [14-17]. However, as cells are complex living materials, thermal fluctuations and cytoskeleton remodeling often enable them to exhibit both solid-like property of elasticity and fluid-like behavior of viscidity. Usually, under such a situation, the material constants of cells cannot be uniquely determined by simply using the elastic constitutive relation in the AFM measurements. For example, it has been reported that the Young's moduli of two types of breast cancer cells exhibit strong dependence on loading rates in the AFM experiments [17].

There have been many studies attempting to determine the viscoelastic property of cells in terms of the traditional systems of Hookean springs and Newtonian viscous dashpots [24, 25]. However, the cytoskeletons of cells are complex polymer networks comprised of polymers with randomly distributed lengths, bending rigidities, orientations and entanglement densities, which usually make the characteristic time scales of creep and relaxation determined from exponential decays widely distributed and infinite in number. We can imagine that the spring-dashpot-type models of viscoelasticity for cells cannot be accurate unless a sufficient number of elements of springs and dashpots are introduced. For example, Susana et al. [24] used a spring-dashpot model with five parameters to fit the indentation curves of MCF-7 cells. Recently, cell-type-specific power-law behaviors have been observed in various types of cultured cells with different measurement techniques when analyzed over certain time scales $[21,26]$. Although such a power-law treatment seems capable of capturing the essential nature of living cells under indentation, it is still far from complete in facilitating unique viscoelastic constitutive relations for general living cells. As has been pointed out by Bu et al. [27], there exist two critical issues in determining the linear constitutive relations of the cells. The first one is that both the behaviors of deformation creep and stress relaxation of a cell should be measured. The second one is that a convolution relation between the measured creep compliance and relaxation modulus should be verified so that whether or not the cell is a linear viscoelastic material can be justified. The reason for such verification is that cells as materials are generally nonlinear, although most of them are nearly linear over certain ranges of some variables of stress, strain, time and temperature. Only after the cells have been confirmed to be linear viscoelastic materials, the constitutive relation and the material constants can be constructed and extracted in terms of the measured creep compliance and relaxation modulus.

In spite of the progresses mentioned above, so far, understanding is still poor in how the constitutive relation of viscoelasticity of each specific type of cells can be precisely determined. In this study, we focus on the measurements of deformation creep and force relaxation trajectories of six different human cell lines by using the technique of AFM indentation. From these trajectories, we determine the creep compliance and relaxation modulus of each cell line by using the classical spring-dashpot and the powerlaw models, respectively. Then, we determine which model gives the better fit and verify whether the determined pair of creep compliance and relaxation modulus satisfy the convolution relation derived from the linear viscoelastic theory, so that we can eventually determine whether or not this cell line is a linear viscoelastic material, and extract the material constants accordingly.

\section{Materials and Methods}

\subsection{Cell Preparation}

The present study focuses on the human normal gastric (GES-1), gastric cancer (SGC7901, NCIN87), normal hepatic (L02), hepatic cancer (HepG2), and hepatic stellate (LX2) cell lines. For the purpose of routine culture, the hepatic cell lines were maintained at $37{ }^{\circ} \mathrm{C}$ in a $5 \% \mathrm{CO} 2$ incubator (Memmert, INE800749L, Germany), and the gastric cell lines were also maintained at the incubator (HealForce, HF90, China) with the same settings. For the AFM indentation, cells harvested from the subculture were seeded on sterilized $35-\mathrm{mm}$ petri dishes which would stay in the incubator for $22-25$ $\mathrm{h}$ prior to each indentation. The medium was changed with normal saline before the AFM indentation to clear extracellular secretion and dead cells.

\subsection{AFM Indentation}

A Nanowizard III BioScience AFM (JPK, Germany) was used for the indentation tests. A modified silicon nitride AFM cantilever (NovaScan, USA) with a spring constant of $0.01 \mathrm{~N} / \mathrm{m}$ was used to indent the cells. A 4.5- $\mu \mathrm{m}$ diameter polystyrene bead was adhered to the cantilever tip. The experiments were conducted at room temperature within $1 \mathrm{~h}$ per dish to ensure the bioactivity of cells. For all the AFM 
indentation measurements, the cantilever was always vertically approaching the nucleus areas of the cells at an ultrafast initial velocity of $50 \mu \mathrm{m} / \mathrm{s}$. Once a pre-defined force for the creep and pre-defined height for the relaxation are reached, the approaching is stopped by controlling the position of cantilever base. Subsequently, under such pre-defined constant force and height, the cantilever was kept in contact with the cells for $20 \mathrm{~s}$, lying within the experimentally observed time range of about 0.01-100 s [28].

\subsection{Deformation Creep and Stress Relaxation of Linear Viscoelastic Materials}

We assume living cells as incompressible linear viscoelastic solids. Then, the corresponding constitutive equations can be written as [29]

$$
\varepsilon_{i j}(t)=\int_{0}^{t} J(t-\tau) \frac{\mathrm{d} \sigma_{i j}(\tau)}{\mathrm{d} \tau} \mathrm{d} \tau, \quad \sigma_{i j}(t)=\int_{0}^{t} Y(t-\tau) \frac{\mathrm{d} \varepsilon_{i j}(\tau)}{\mathrm{d} \tau} \mathrm{d} \tau
$$

where $J(t)$ is the creep compliance of the viscoelastic body, $Y(t)$ the relaxation modulus, $\varepsilon_{i j}$ and $\sigma_{i j}$ are components of the strain and stress tensors, respectively. Applying the Laplace transform to Eq. (1) in terms of time, we obtain

$$
\hat{\varepsilon}_{i j}=s \hat{J}(s) \hat{\sigma}_{i j}(s), \quad \hat{\sigma}_{i j}=s \hat{Y}(s) \hat{\varepsilon}_{i j}(s)
$$

where $\hat{J}(s), \hat{Y}(s), \hat{\varepsilon}_{i j}(s)$ and $\hat{\sigma}_{i j}(s)$ are the Laplace transforms of $J(t), Y(t), \varepsilon_{i j}$ and $\sigma_{i j}$. From Eq. $(2)$, we can easily deduce that $\hat{Y}(s)=1 / s^{2} \hat{J}(s)$, which corresponds to the following relation in real time space as [29]

$$
\int_{0}^{t} J(t-\tau) Y(t) \mathrm{d} \tau=t
$$

We note that Eq. (3) is derived based on the assumption of incompressible linear viscoelasticity. In the following sections, we will use this relation as the criterion to verify whether the mechanical properties of a living cell are linear, so that one can know whether or not the experimentally determined creep compliance and relaxation modulus can be used to construct the constitutive relation.

In practical applications of viscoelastic modeling, there exist many different models to specify the creep compliance and relaxation modulus in Eq. (1). Traditionally, the constitutive relation of a general linear viscoelastic solid can be represented by a network of linear combinations of springs and dashpots. Following such a spring-dashpot representation, the creep compliance and relaxation modulus can be expressed though a so-called Prony series [30, 31],

$$
J(t)=\frac{1}{E}\left[1+\sum_{i=0}^{m} c_{i}\left(1-\mathrm{e}^{-t / \tau_{i}}\right)\right], Y(t)=E\left[1-\sum_{j=0}^{n} d_{j}\left(1-\mathrm{e}^{-t / \tau_{j}^{\prime}}\right)\right]
$$

where $E$ represents the initial Young's modulus, $\tau_{i}, \tau_{j}^{\prime}$ are the typical creep and relaxation time scales, $m$ and $n$ together are related to the number of springs and dashpots in the system. The Prony series in Eq. (4) are named as the $m$-th order and $n$-th order in this study, respectively.

We can see from Eq. (4) that the spring-dashpot type constitutive relations of the viscoelastic materials usually consist of a finite number of scales of the characteristic times for the creep and relaxation behaviors. If a large number of terms of Eq. (4) are required in accurately describing the mechanical behaviors of the materials, e.g., the living cells, then the expressions of Eq. (4) may become no longer practical as the coefficient of each term can be very hard to uniquely determine. However, like the soft glass rheology theory derived from soft matter physics, the viscoelastic spectrum of living cells lacks any distinct timescales that can be identified with discrete structural elements or processes, meaning that a large number of parameters will be inevitably involved to fit experimental measurements successfully by using Eq. (4). In order to resolve this problem, we adopt the power-law-type creep function as follows $[29,32]$

$$
J(t)=\frac{1}{E}\left(t / \tau_{0}\right)^{\beta}
$$

where $E$ still represents the effective Young's modulus at time $\tau_{0}$, then $1 / E$ becomes the elastic compliance, and $\beta$ characterizes the degree of dissipation or "fluidity" of the viscoelastic material [3234]. When $\beta$ approaches zero, Eq. (5) becomes the inverse of material stiffness, corresponding to the 


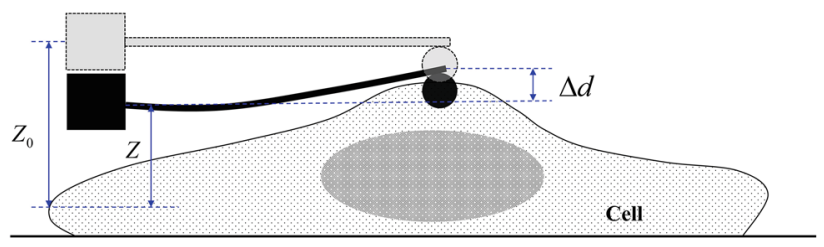

Fig. 1. Schematic of the experimental setup for the indentation of a cell using spherical indenter probes

deformation of a purely elastic solid. On the contrary, if $\beta$ tends to unity, Eq. (5) becomes corresponding to the deformation of a purely viscous Newtonian fluid. For living cells, the measured $\beta$ are typically located between 0.1 and 0.5 [32-34]. For incompressible linear viscoelastic materials, the relation in Eq. (3) must be satisfied. Then, the corresponding relaxation modulus can be determined from Eq. (3) as follows $[27]$

$$
Y(t)=\frac{E}{\Gamma(1+\beta) \Gamma(1-\beta)}\left(t / \tau_{0}\right)^{-\beta}
$$

In the following sections, we will use the classical spring-dashpot model represented by Eq. (4), and the power-law model by Eqs. (5) and (6) to fit the experimental measurements on the creep and relaxation behaviors.

\subsection{AFM Measurements and Data Processing}

Figure 1 shows the process of indentation of a single cell, where $Z$ is the vertical height of the probe base, $Z_{0}$ the value of $Z$ at the contact point, $d$ the vertical deflection of the cantilever. In addition, we define $\delta$ as the indentation depth and $P$ the indentation force. If the spring constant of the cantilever is $k$, then we have

$$
P=k \Delta d
$$

where

$$
\delta=Z_{0}-Z-\Delta d, \quad \Delta d=d-d_{0}
$$

When the incompressible viscoelastic solid is indented by a rigid sphere with radius $R$, the relation between the indentation force and indentation depth can be expressed as [35-37]

$$
P(t)=\frac{8 \sqrt{R}}{3} \int_{0}^{t} Y(t-\tau) \frac{\partial\left[\delta^{3 / 2}(\tau)\right]}{\partial \tau} \mathrm{d} \tau
$$

or

$$
\delta^{3 / 2}(t)=\frac{3}{8 \sqrt{R}} \int_{0}^{t} J(t-\tau) \frac{\partial P(\tau)}{\partial \tau} \mathrm{d} \tau
$$

When a step external force or displacement is applied during the indentation, we have $P(t)=P_{0} H(t)$ and $\delta(t)=\delta_{0} H(t)$. Then, Eqs. (9) and (10) become [27,35]

$$
J(t)=\frac{8 \sqrt{R}}{3 P_{0}} \delta(t)^{3 / 2} \text { and } Y(t)=\frac{3}{8 \sqrt{R} \delta_{0}^{3 / 2}} P(t)
$$

Figure 2 shows the time trajectories during the displacement creep and force relaxation experiments for the AFM indentation of normal hepatic cells. Figure 2a illustrates the creep test, in which the predefined indentation force is sustained at $0.88 \mathrm{nN}$ for $20 \mathrm{~s}$ after the rapid loading and before unloading, while the Z-position decreases from $11.89 \mu \mathrm{m}$ to $5.88 \mu \mathrm{m}$ with time over $20 \mathrm{~s}$. Figure $2 \mathrm{~b}$ demonstrates the relaxation test, in which the Z-position is kept at $6.91 \mu \mathrm{m}$ after the rapid loading and before unloading, while the indentation force decreases from $1.9 \mathrm{nN}$ to $0.7 \mathrm{nN}$ over $20 \mathrm{~s}$. 

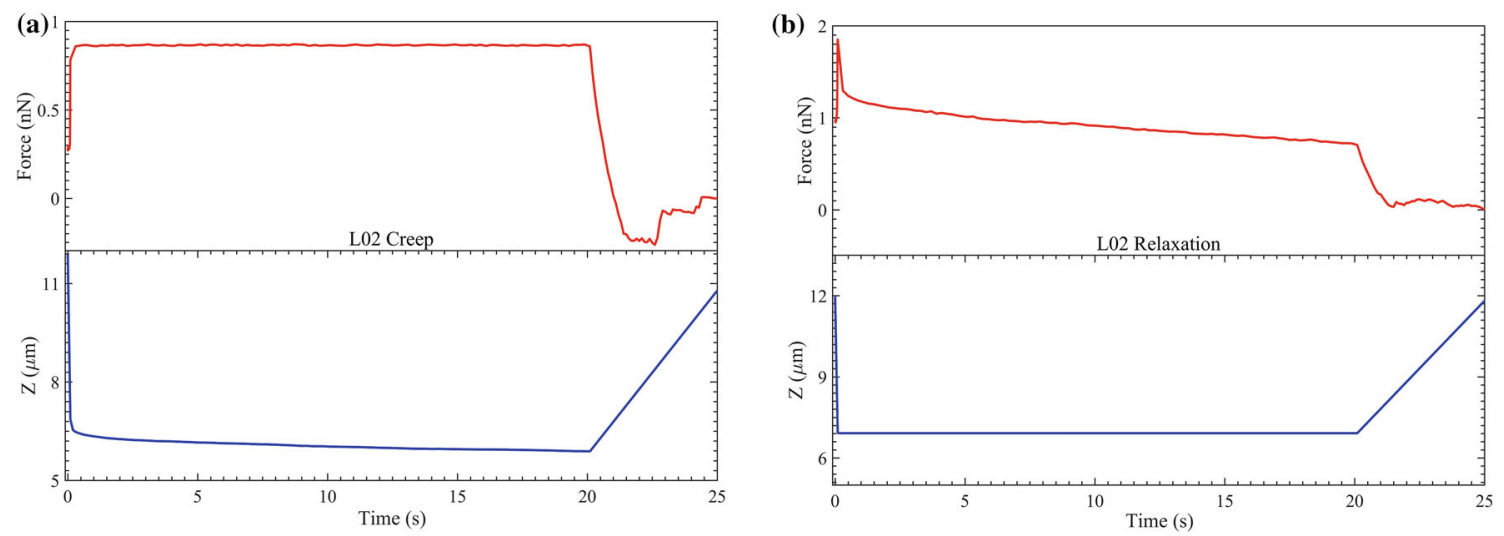

Fig. 2. Creep and relaxation measurements on normal hepatic cells (L02): a creep of the Z-position of cantilever base under force-controlled loading; $\mathbf{b}$ relaxation of the indentation force under displacement-controlled loading

(a)

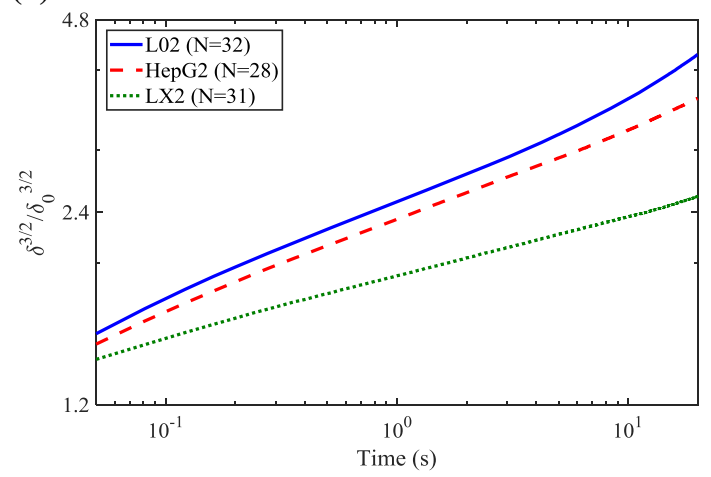

(c)

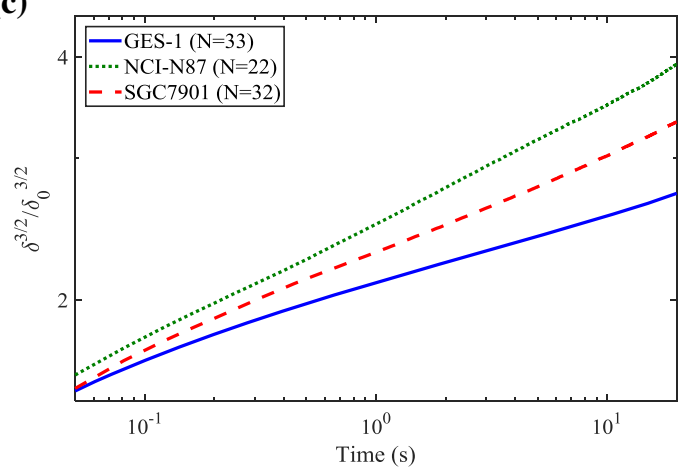

(b)

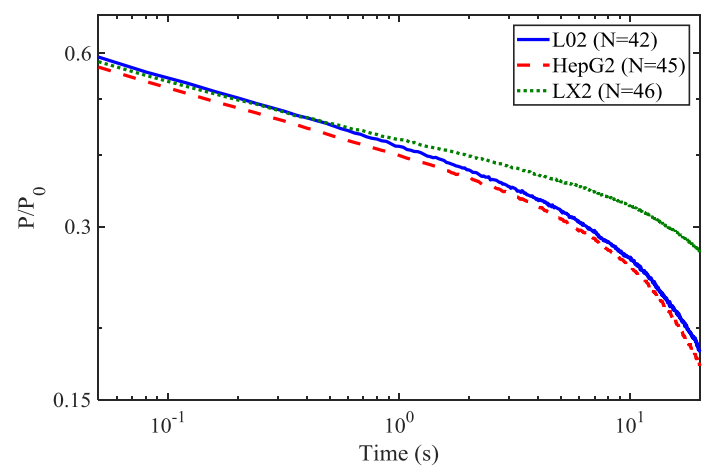

(d)

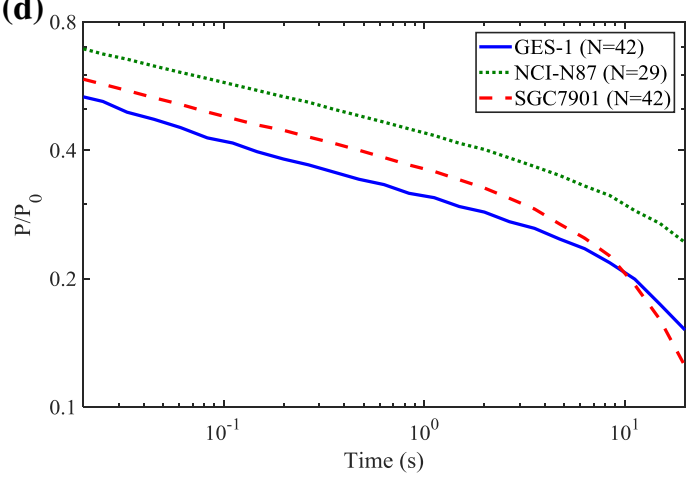

Fig. 3. Normalized indentation depth of a human hepatic cell lines and $\mathbf{c}$ human gastric cell lines, and normalized indentation force of $\mathbf{b}$ human hepatic cell lines and $\mathbf{d}$ human gastric cell lines, where $N$ is the number of cells used for the measurements

\section{Result and Discussion}

We repeated the deformation creep and force relaxation tests for $N$ times by using $N$ different cells for each cell line. During each test, we recorded the indentation depth $\delta(t)$ for the creep test, and the indentation force $P(t)$ for the relaxation test, as a function of time. We performed ensemble average over $N$ different trajectories to obtain the mean value of the normalized indentation depth of the $3 / 2$ power, $\left\langle\left(\delta / \delta_{0}\right)^{3 / 2}\right\rangle$, and the normalized indentation force, $\left\langle P / P_{0}\right\rangle$. Figure 3 shows the comparison on $\left\langle\left(\delta / \delta_{0}\right)^{3 / 2}\right\rangle$ and $\left\langle P / P_{0}\right\rangle$ as functions of time for the hepatic $(\mathrm{a}, \mathrm{b})$ and gastric cells $(\mathrm{c}, \mathrm{d})$. It can be 
(a)

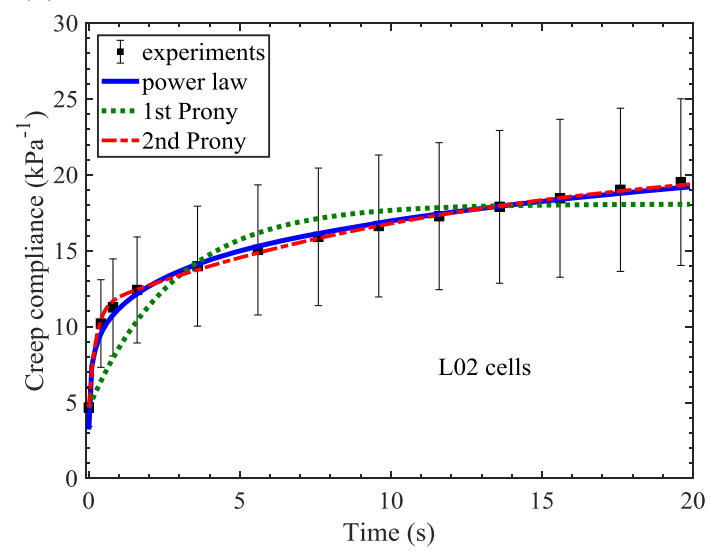

(c)

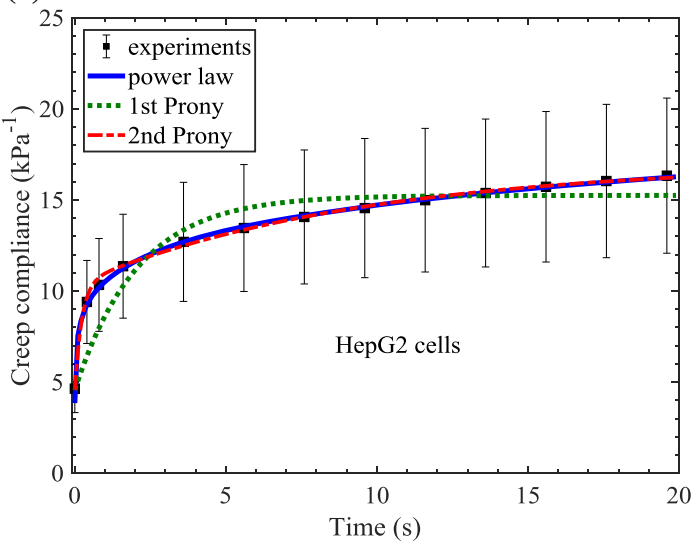

(e)

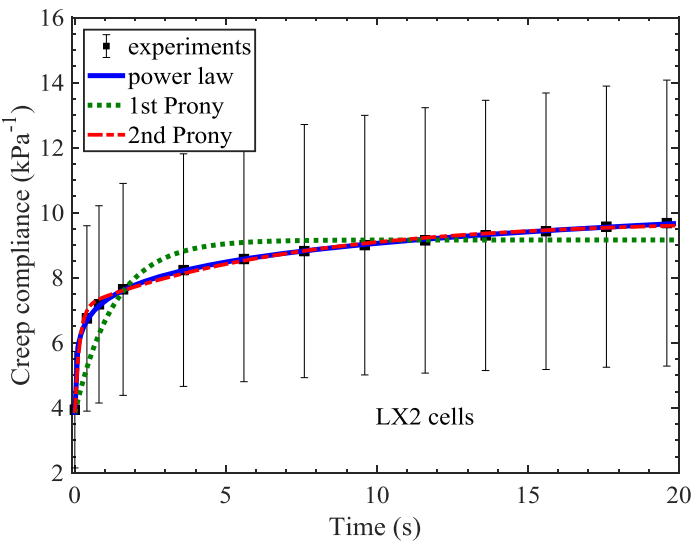

(b)

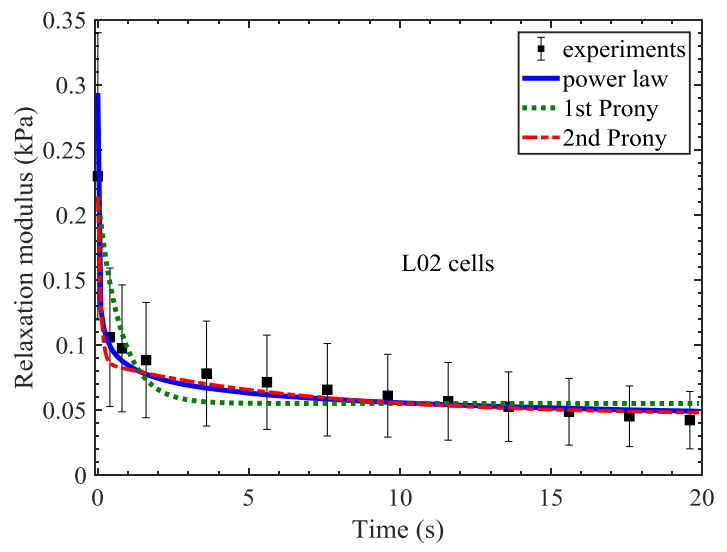

(d)

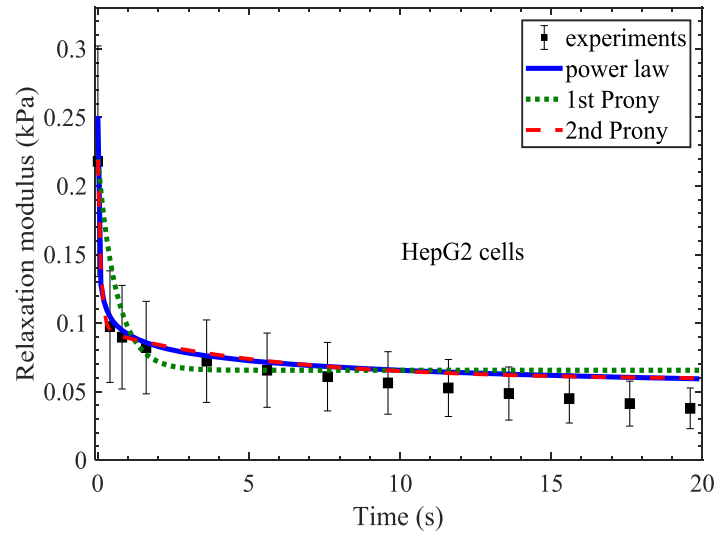

(f)

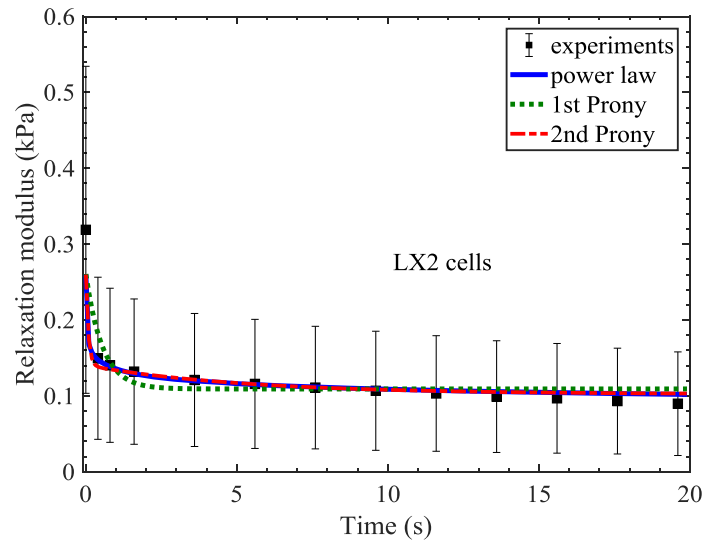

Fig. 4. Comparison of experimental results and the corresponding least-square fittings in terms of the power-law and spring-dashpot models for the creep compliance of a L02, $\mathbf{c}$ HepG2, and e LX2, and the relaxation modulus of b L02, d HepG2, and f LX2

seen from Fig. 3a, b that the L02 and HepG2 cells show similar fluidity, while the LX2 cells appear more solid-like than them. This is not surprise since LX2 cells are the major cells involved in liver fibrosis, which is the formation of scar tissue in response to liver damage. However, for the gastric cell lines in Fig. 3c, d, the creep and relaxation results give contradictory predictions. For example, Fig. 3c indicates that the fluidity of the three gastric cell lines is in the order of NCI-N87 > SGC7901 > GES-1 according to the creep test, while Fig. 3d shows an opposite trend in terms of the relaxation test. 
(a)

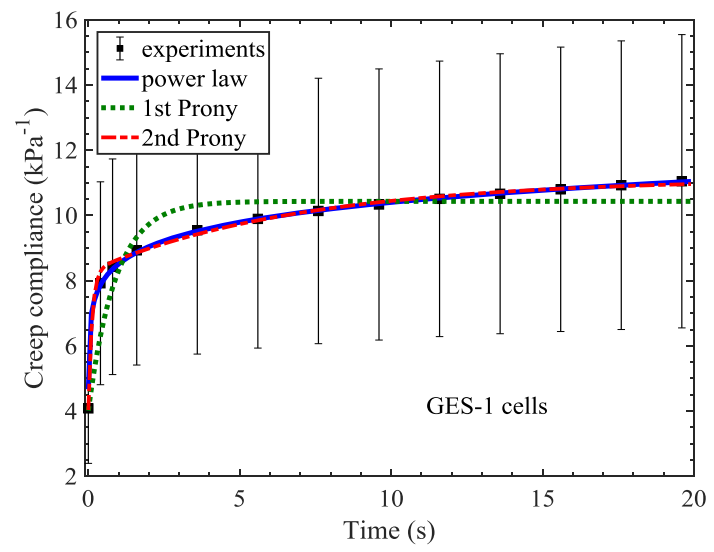

(c)

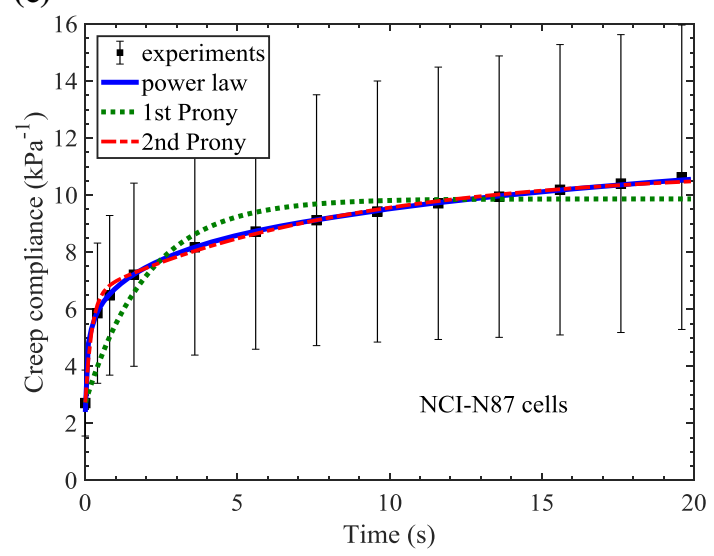

(e)

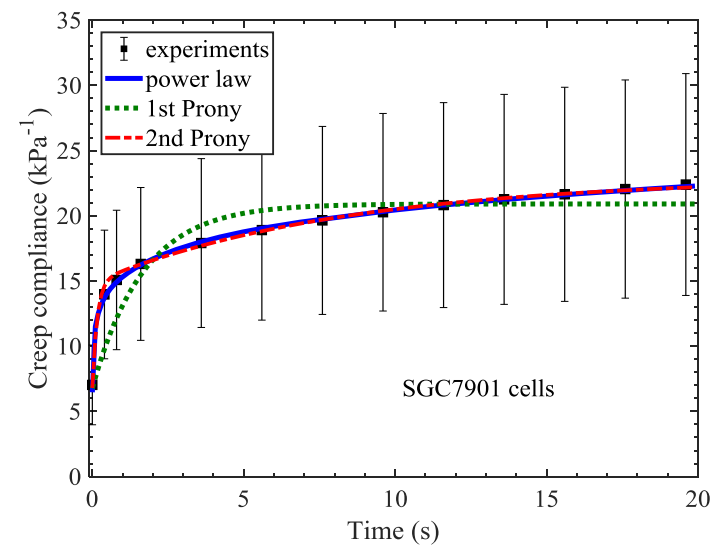

(b)

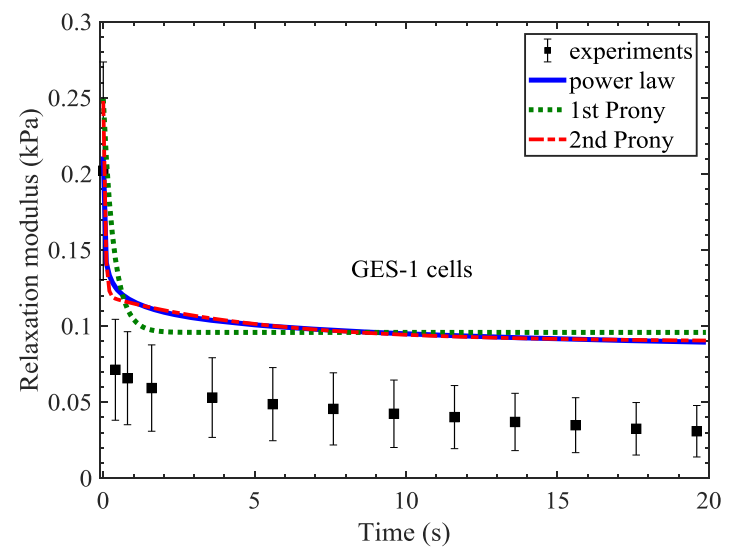

(d)

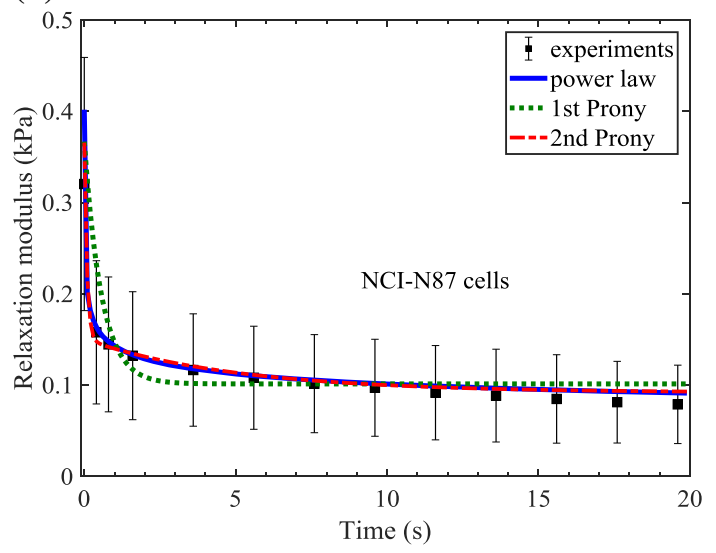

(f)

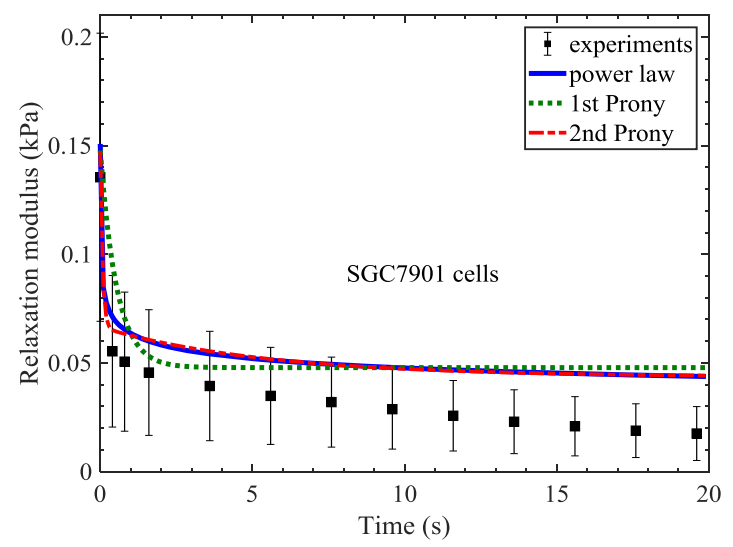

Fig. 5. Comparison of experimental results and the corresponding least-square fittings in terms of the power-law and spring-dashpot models for the creep compliance of a GES-1, c NCI-N87, and e SGC7901, and the relaxation modulus of b GES-1, d NCI-N87, and f SGC7901

These unreasonable results imply that at least two of the three cell lines are nonlinear materials, leading to wrong predictions of mechanical properties based on the linear theory. This fact indicates that one cannot simply use the experimental results of either creep or relaxation to describe the mechanical properties of living cells without verifying whether the creep compliance and relaxation modulus satisfy the linearity requirement in Eq. (3). 
Table 1. Least-square fitting parameters for the power-law model, $e=1 \times 10^{-3}$

\begin{tabular}{llll}
\hline & $1 / E\left(\mathrm{kPa}^{-1}\right)$ & $\tau_{0}(\mathrm{~s})$ & $\beta$ \\
\hline L02 & $4.643 \pm 2 e$ & $7.586 e \pm 0.02 e$ & $0.1804 \pm 0.4 e$ \\
HepG2 & $4.539 \pm e$ & $5.808 e \pm 0.25 e$ & $0.1867 \pm 1.1 e$ \\
LX2 & $3.826 \pm e$ & $1.074 e \pm 0.03 e$ & $0.1247 \pm 0.4 e$ \\
GES-1 & $3.997 \pm e$ & $0.1674 e \pm 0.0019 e$ & $0.08696 \pm 0.09 e$ \\
NCI-N87 & $2.717 \pm e$ & $2.3 e \pm 0.012 e$ & $0.1497 \pm 0.1 e$ \\
SGC7901 & $6.744 \pm e$ & $1.4 e \pm 0.011 e$ & $0.125 \pm 0.1 e$ \\
\hline
\end{tabular}

Table 2. Least-square fitting parameters for the spring-dashpot model in terms of the first-order Prony series, $e=1 \times 10^{-3}$

\begin{tabular}{llll}
\hline & $1 / E\left(\mathrm{kPa}^{-1}\right)$ & $c_{1}$ & $\tau_{1}(\mathrm{~s})$ \\
\hline L02 & $4.658 \pm 7 e$ & $2.881 \pm 8 e$ & $2.87 \pm 0.035$ \\
HepG2 & $4.552 \pm 6 e$ & $2.35 \pm 5 e$ & $2.085 \pm 0.027$ \\
LX2 & $3.834 \pm e$ & $1.389 \pm 3 e$ & $1.316 \pm 0.019$ \\
GES-1 & $4.003 \pm 4 e$ & $1.606 \pm 3 e$ & $0.9042 \pm 0.0149$ \\
NCI-N87 & $2.723 \pm 4 e$ & $2.624 \pm 5 e$ & $2.054 \pm 0.025$ \\
SGC7901 & $6.761 \pm 8 e$ & $2.092 \pm 4 e$ & $1.676 \pm 0.023$ \\
\hline
\end{tabular}

Table 3. Least-square fitting parameters for the spring-dashpot model in terms of the second-order Prony series, $e=1 \times 10^{-3}$

\begin{tabular}{llllll}
\hline & $1 / E\left(\mathrm{kPa}^{-1}\right)$ & $c_{1}$ & $\tau_{1}(\mathrm{~s})$ & $c_{2}$ & $\tau_{2}(\mathrm{~s})$ \\
\hline L02 & $4.644 \pm e$ & $1.426 \pm 3 e$ & $0.2252 \pm 2.5 e$ & $2.249 \pm 8 e$ & $13.06 \pm 0.12$ \\
HepG2 & $4.541 \pm e$ & $1.281 \pm 3 e$ & $0.2275 \pm 2.6 e$ & $1.471 \pm 4 e$ & $9.375 \pm 0.079$ \\
LX2 & $3.826 \pm e$ & $0.848 \pm 2 e$ & $0.1521 \pm 2 e$ & $0.7065 \pm 1.9 e$ & $7.278 \pm 0.064$ \\
GES-1 & $3.997 \pm e$ & $1.07 \pm 2 e$ & $0.121 \pm 2 e$ & $0.7137 \pm 2 e$ & $6.979 \pm 0.065$ \\
NCI-N87 $^{*}$ & $2.716 \pm e$ & $1.4 \pm 4 e$ & $0.2092 \pm 3 e$ & $1.609 \pm 4 e$ & $8.388 \pm 0.075$ \\
SGC7901 $^{*}$ & $6.744 \pm e$ & $1.205 \pm 3 e$ & $0.1585 \pm 3 e$ & $1.194 \pm 3 e$ & $8.318 \pm 0.063$ \\
\hline
\end{tabular}

In order to predict the creep compliance and relaxation modulus from the indentation test for different cell lines, we consider the ensemble average on both sides of Eq. (11), which gives

$$
\langle J(t)\rangle=\left\langle\frac{8 \sqrt{R}}{3 P_{0}} \delta(t)^{3 / 2}\right\rangle \text { and }\langle Y(t)\rangle=\left\langle\frac{3}{8 \sqrt{R} \delta_{0}^{3 / 2}} P(t)\right\rangle
$$

The solid squares in Figs. 4 and 5 show the experimental results on the average values of creep compliance and relaxation modulus as functions of time for both hepatic (Fig. 4) and gastric (Fig. 5) cells. These experimental data can be fitted by the classical spring-dashpot model and the power-lawtype model, as shown in Eqs. (4-6). By using the least-square fitting method, Tables 1, 2 and 3 list the fitting parameters of the power-law and spring-dashpot models in terms of the creep experiments. Interestingly, the results of fitted effective elastic compliance $1 / E$ for all the cell lines are almost not affected by the model selection. In contrary, values of the fitted characteristic time scales, $\tau_{i}$, in terms of the spring-dashpot model, even exhibit differences in orders of magnitudes. Further studies are therefore needed for understanding the relation between such large discrepancies and the corresponding biological appearances of cells.

Figures 4a, c, e and 5a, c, e show the comparison between the experimental results on creep compliance and the least-square fittings of the experimental data by using the power-law and spring-dashpot models in terms of the $1^{\text {st }}$-order and 2 nd-order Prony series with the fitting parameters listed in Tables 1, 2 and 3. It can be seen from Figs. 4a, c, e and 5a, c, e that the 3-parameter power-law model can fit all the experimental results very well, the fitting results based on the 3-parameter spring-dashpot model show large discrepancies with experiments, and those based on the 5-parameter spring-dashpot model seem close to the experiments. However, we can see from Figs. 4a, c, e and 5a, c, e that the 
(a)

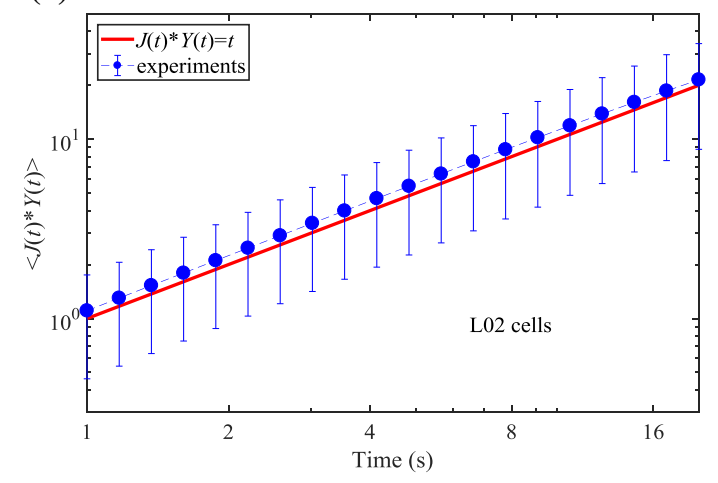

(c)

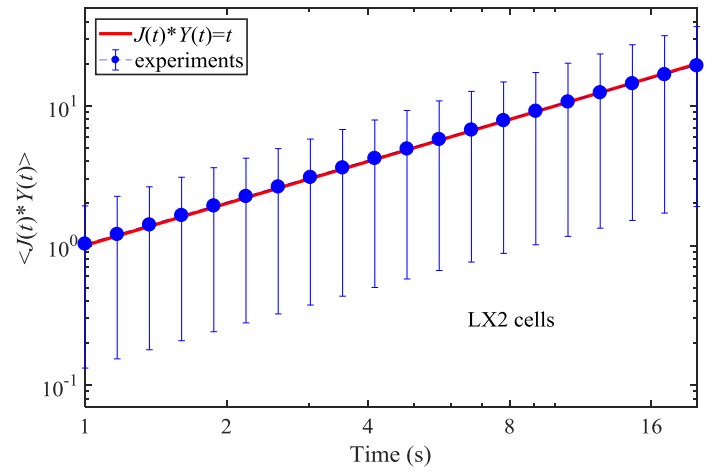

(e)

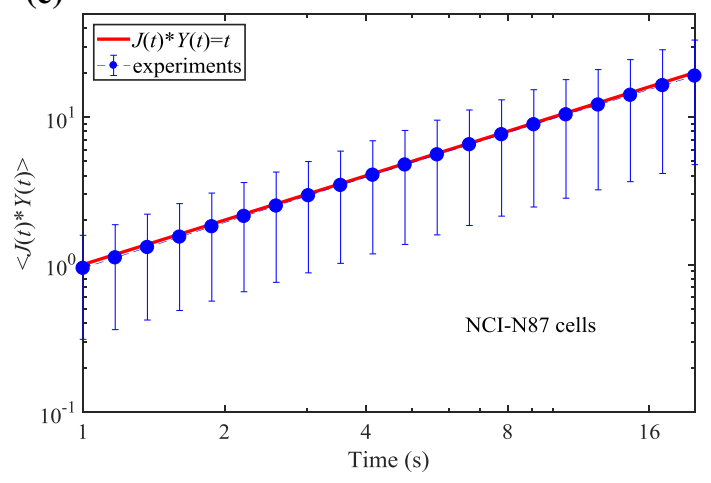

(b)

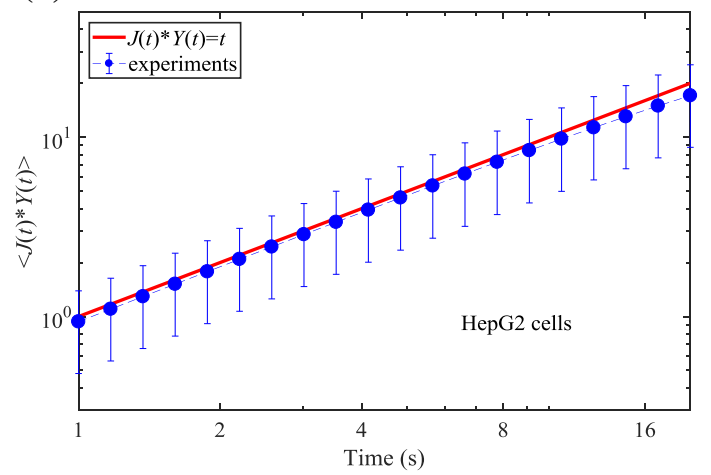

(d)

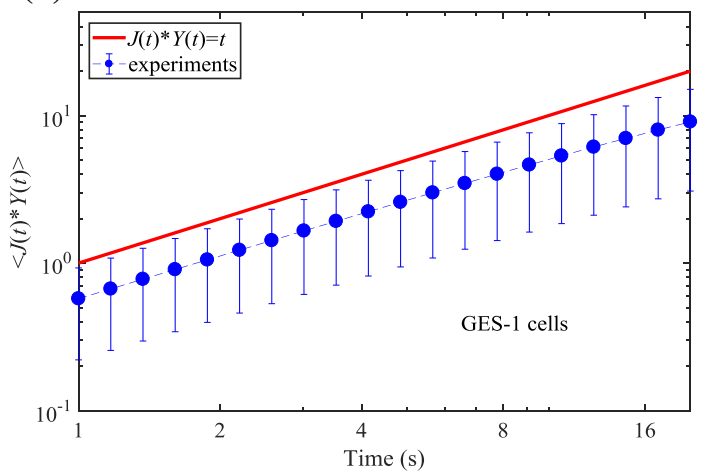

(f)

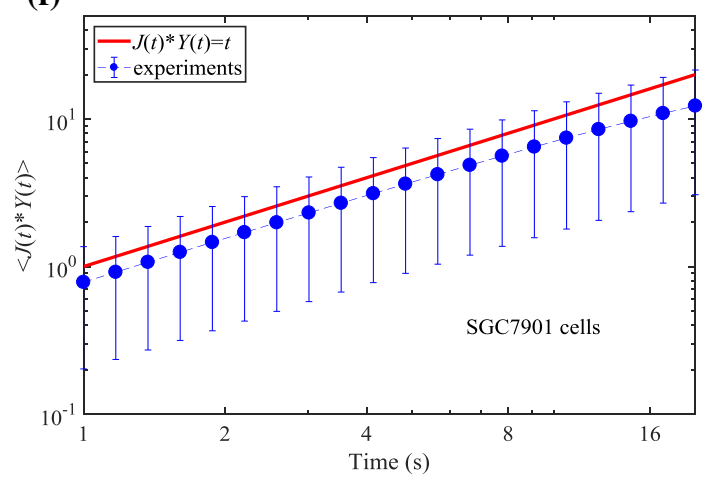

Fig. 6. Convolution of the experimentally measured creep compliance and relaxation modulus of a L02, b HepG2, c LX2, d GES-1, e NCI-N87, and f SGC7901 cells

fitting results based on the 5-parameter spring-dashpot model show unrealistic sharp turning points or large slops at times close to the corresponding time scales $\tau_{1}$ in Table 3 for each cell line.

Once the creep compliance is obtained by fitting the creep experiments, the relaxation modulus is obtained by applying the fitting parameters in Tables 1, 2 and 3 to Eqs. (4-6). Figures 4b, d, f and 5b, $\mathrm{d}$, f show the comparison between the experimental results on relaxation modulus and the theoretical predictions based on the power-law and spring-dashpot models in terms of the fitting parameters in Tables 1, 2 and 3. It can be seen from Figs. 4b, d, f and 5b, d, f that the theoretical predictions agree with the experiments very well for cell lines of L02, HepG2, LX2 and NCI-N87, but not for GES-1 and SGC7901. This comparison implies that L02, HepG2, LX2 and NCI-N87 are linear viscoelastic materials, and GES-1 and SGC7901 are nonlinear. 
In order to further verify which cell line is linear or nonlinear, we consider the derived relation in Eq. (3). Once we have experimentally obtained the creep compliance and the relaxation modulus as functions of time, $J(t)$ and $Y(t)$, we can numerically calculate their convolution, $J(t) * Y(t)$, then perform ensemble average to obtain $\langle J(t) * Y(t)>$. We compare the experimentally determined $\langle J(t) * Y(t)\rangle$ with the function $t$. The linear viscoelasticity of a material needs these two functions to be identical. Otherwise, the material must be nonlinear. Figure 6 shows such comparisons. We can see from Fig. 6 that the convolutions, $\langle J(t) * Y(t)\rangle$, for the cell lines of L02, HepG2, LX2 and NCI-N87 are close to the function $t$, but those for GES-1 and SGC7901 show obvious discrepancies. In order to give a quantitative estimation on such a discrepancy, we calculate $(\langle J(t) * Y(t)>$ rlanglet $) / t$ for each cell line. The values are $7 \%$ to $13.1 \%$ for L02, $-14.6 \%$ to $-4.8 \%$ for HepG2, $-2.8 \%$ to $2.7 \%$ for LX2, and $-5.5 \%$ to $2 \%$ for NCI-N87. However, such a value becomes $-54.7 \%$ to $-42.6 \%$ for GES- 1 , and $-38.7 \%$ to $-21.7 \%$ for SGC7901. Therefore, it can be easily deduced that the cell lines of NCI-N87 and GES-1 show obvious nonlinear material properties. For these two cell lines, one cannot simply use the linear viscoelastic constitutive relation to predict their mechanical behaviors.

\section{Conclusions}

In summary, we have used the AFM to measure the deformation creep and force relaxation trajectories of six different human cell lines. Based on these measurements, we have determined the creep compliance and relaxation modulus of these cells by the technique of least-square fitting in terms of the power-law and spring-dashpot models, respectively. We found that the 3-parameter power-law model can fit the experiments very well. For the spring-dashpot model, only when the fitting parameters are at least 5, then the fitting results can be acceptable, but there still exist unexpected sharp turns in the fitting curves. We have further verified whether the measured creep compliance and relaxation modulus satisfy the convolution relation derived from the linear viscoelastic theory, by which one can know whether a cell line follows a linear constitutive relation. We found that the human normal hepatic (L02), hepatic cancer (HepG2), hepatic stellate (LX2) and gastric cancer (NCI-N87) cell lines are linear viscoelastic materials, and human normal gastric (GES-1) and gastric cancer (SGC7901) cell lines are nonlinear. The obtained fitting parameters can be used as the corresponding material constants for the former, but not the latter. Not only the material constants, for the cell lines with nonlinear mechanical properties, like GES-1 and SGC7901, the simple creep and relaxation behaviors can even give contradictory predictions on their mechanical properties and behaviors.

Acknowledgements. We acknowledge the Institute of Pathology, School of Basic Medical Sciences, Lanzhou University, for providing cells. This study is supported by grants from the National Natural Science Foundation of China (11472119, 11602099) and the 111 Project (B14044).

Open Access This article is distributed under the terms of the Creative Commons Attribution 4.0 International License (http://creativecommons.org/licenses/by/4.0/), which permits unrestricted use, distribution, and reproduction in any medium, provided you give appropriate credit to the original author(s) and the source, provide a link to the Creative Commons license, and indicate if changes were made.

\section{References}

[1] Lautenschläger F, Paschke S, Schinkinger S, Bruel A, Beil M, Guck J. The regulatory role of cell mechanics for migration of differentiating myeloid cells. Proc. Natl. Acad. Sci. USA. 2009;106(37):15696-701.

[2] Nelson CM, Jean RP, Tan JL, Liu WF, Sniadecki NJ, Spector AA, Chen CS. Emergent patterns of growth controlled by multicellular form and mechanics. Proc. Natl. Acad. Sci. USA. 2005;102(33):11594-9.

[3] Qian J, Wang JZ, Gao HJ. Lifetime and strength of adhesive molecular bond clusters between elastic media. Langmuir. 2008;24(4):1262-70.

[4] Kumar S, Maxwell IZ, Heisterkamp A, Polte TR, Lele TP, Salanga M, Mazur E, Ingber DE. Viscoelastic retraction of single living stress fibers and its impact on cell shape, cytoskeletal organization, and extracellular matrix mechanics. Biophys. J. 2006;90(10):3762-73.

[5] Huang C, Butler PJ, Tong S, Muddana HS, Bao G, Zhang S. Substrate stiffness regulates cellular uptake of nanoparticles. Nano Lett. 2013;13(4):1611-5.

[6] Wang JZ, Li L. Coupled elasticity-diffusion model for the effects of cytoskeleton deformation on cellular uptake of cylindrical nanoparticles. J. R. Soc. Interface. 2015;12(102):20141023.

[7] Wang JZ, Li L, Zhou YH. Creep effect on cellular uptake of viral particles. Sci. Bull. 2014;59(19):2277-81. 
[8] Fuhrmann A, Staunton JR, Nandakumar V, Banyai N, Davies PCW, Ros R. AFM stiffness nanotomography of normal, metaplastic and dysplastic human esophageal cells. Phys. Biol. 2011;8(1):015007.

[9] Prabhune M, Belge G, Dotzauer A, Bullerdiek J, Radmacher M. Comparison of mechanical properties of normal and malignant thyroid cells. Micron. 2012;43(12):1267-72.

[10] Efremov YM, Lomakina ME, Bagrov DV, Makhnovskiy PI, Alexandrova AY, Kirpichnikov MP, Shaitan KV. Mechanical properties of fibroblasts depend on level of cancer transformation. BBA-Mol. Cell Res. 2014;1843(5):1013-9.

[11] Maciaszek JL, Andemariam B, Lykotrafitis G. Microelasticity of red blood cells in sickle cell disease. J. Strain Anal. Eng. 2011;46(5):368-79.

[12] Lim CT. Single cell mechanics study of the human disease malaria. Biomech. Sci. Eng. 2006;1(1):82-92.

[13] Lee GYH, Lim CT. Biomechanics approaches to studying human diseases. Trends. Biotech. 2007;25(3):1118.

[14] Lekka M, Laidler P, Ignacak J, Łabedź M, Lekki J, Struszczyk H, Stachura Z, Hrynkiewicz AZ. The effect of chitosan on stiffness and glycolytic activity of human bladder cells. BBA-Mol. Cell Res. 2001; 1540(2):127-36.

[15] Lekka M, Gil D, Pogoda K, Dulińska-Litewka J, Jach R, Gostek J, Klymenko O, Prauzner-Bechcicki S, Stachura Z, Wiltowska-Zuber J, Okoń K, Laidler P. Cancer cell detection in tissue sections using AFM. Arch. Biochem. Biophys. 2012;518(2):151-6.

[16] Lekka M, Pogoda K, Gostek J, Klymenko O, Prauzner-Bechcicki S, Wiltowska-Zuber J, Jaczewska J, Lekki J, Stachura Z. Cancer cell recognition-mechanical phenotype. Micron. 2012;43(12):1259-66.

[17] Li QS, Lee GY, Ong CN, Lim CT. AFM indentation study of breast cancer cells. Biochem. Biophys. Res. Commun. 2008;374(4):609-13.

[18] Wang H, Wilksch JJ, Strugnell RA, Gee ML. Role of capsular polysaccharides in biofilm formation: an AFM nanomechanics study. ACS. Appl. Mater. Interface. 2015;7(23):13007-13.

[19] Puig-De-Morales M, Grabulosa M, Alcaraz J, Mullol J, Maksym GN, Fredberg JJ, Navajas D. Measurement of cell microrheology by magnetic twisting cytometry with frequency domain demodulation. J. Appl. Physiol. 2001;91(3):1152-9.

[20] Titushkin I, Cho M. Distinct membrane mechanical properties of human mesenchymal stem cells determined using laser optical tweezers. Biophys. J. 2006;90(7):2582-91.

[21] Desprat N, Richert A, Simeon J, Asnacios A. Creep function of a single living cell. Biophys. J. 2005;88(3):2224-33.

[22] Yamada S, Wirtz D, Kuo SC. Mechanics of living cells measured by laser tracking microrheology. Biophys. J. 2000;78(4):1736-47.

[23] Crocker JC, Valentine MT, Weeks ER, Gisler T, Kaplan PD, Yodh AG, Weitz DA. Two-point microrheology of inhomogeneous soft materials. Phys. Rev. Lett. 2000;85(4):888.

[24] Moreno-Flores S, Benitez R, dM Vivanco M, Toca-Herrera JL. Stress relaxation and creep on living cells with the atomic force microscope: a means to calculate elastic moduli and viscosities of cell components. Nanotechnology. 2010;21(44):445101.

[25] Moreno-Flores S, Benitez R, dM Vivanco M, Toca-Herrera JL. Stress relaxation microscopy: imaging local stress in cells. J. Biomech. 2010;43(2):349-54.

[26] Schierbaum N, Rheinlaender J, Schäffer TE. Viscoelastic properties of normal and cancerous human breast cells are affected differently by contact to adjacent cells. Acta Biomater. 2017;55:239-48.

[27] $\mathrm{Bu} \mathrm{Y,} \mathrm{Li} \mathrm{L,} \mathrm{Wang} \mathrm{JZ.} \mathrm{Power} \mathrm{law} \mathrm{creep} \mathrm{and} \mathrm{relaxation} \mathrm{with} \mathrm{the} \mathrm{atomic} \mathrm{force} \mathrm{microscope:} \mathrm{determining}$ viscoelastic property of living cells. Sci. China Technol. Sc. 2019;62(5):781-6.

[28] Fabry B, Maksym GN, Butler JP, Glogauer M, Navajas D, Taback NA, Millet EJ, Fredberg JJ. Time scale and other invariants of integrative mechanical behavior in living cells. Phys. Rev. E. 2003;68(4):041914.

[29] Findley WN, Lai JS, Onaran K. Creep and relaxation of nonlinear viscoelastic materials: with an introduction to linear viscoelasticity. In: Linear viscoelastic constitutive equations. North-Holland: Journal of Applied Mechanics; 1976. pp. 50-107.

[30] Christensen R. Theory of viscoelasticity: an introduction. In: Viscoelastic stress stain constitutive relations. London: Academic Press; 1982. pp. 1-34.

[31] Cao YP, Ji XY, Feng XQ. Geometry independence of the normalized relaxation functions of viscoelastic materials in indentation. Philos. Mag. 2010;90(12):1639-55.

[32] Kollmannsberger P, Fabry B. Linear and nonlinear rheology of living cells. Rev. Mater. Res. 2011;41:75-97.

[33] Maloney JM, Nikova D, Lautenschläger F, Clarke E, Langer R, Guck J, Van Vliet KJ. Mesenchymal stem cell mechanics from the attached to the suspended state. Biophys. J. 2010;99(8):2479-87.

[34] Cai P, Mizutani Y, Tsuchiya M, Maloney JM, Fabry B, Van Vliet KJ, Okajima T. Quantifying cell-to-cell variation in power-law rheology. Biophys. J. 2013;105(5):1093-102. 
[35] Oyen ML. Spherical indentation creep following ramp loading. J. Mater. Res. 2005;20(8):2094-100.

[36] Lee EH, Radok JRM. The contact problem for viscoelastic bodies. J. Appl. Mech. 1960;27(3):438-44.

[37] Johnson KL. Contact mechanics. In: Normal contact of inelastic solids. Cambridge: Cambridge University Press; 1985. pp. 153-96. 